\title{
Erratum to: Theory, experiment and applications of metamaterials [Sci China-Phys Mech Astron, 2015, 58: 127001]
}

\author{
WenXuan Tang ${ }^{1 \dagger \dagger}$, ZhongLei Mei ${ }^{2 \dagger \dagger}$, and TieJun Cui ${ }^{1 *}$ \\ ${ }^{1}$ State Key Laboratory of Millimeter Waves, Southeast University, Nanjing 210096, China; \\ ${ }^{2}$ School of Information Science and Engineering, Lanzhou University, Lanzhou 730000, China
}

Citation: W. X. Tang, Z. L. Mei, and T. J. Cui, Erratum to: Theory, experiment and applications of metamaterials [Sci China-Phys Mech Astron, 2015, 58: 127001], Sci. China-Phys. Mech. Astron. 59, 624201 (2016), doi: 10.1007/s11433-015-5770-8

In the original publication of this paper, the captions of Figures 4, 5 and 9 were documented incorrectly. The references numbers were missing [1]. The corrected information is listed as below:

"Figure 4 (Color online) The first prototype of NIM metamaterial [4]."

"Figure 5 (Color online) The first 2D cloak at microwave frequencies with a plot of the material parameters that are implemented [49]."

"Figure 9 (Color online) The radiation of a line source in (a) free space and (b) anisotropic metamaterial with the permeability component $\mu_{y} \rightarrow 0$. Fabricated anisotropic zero-index metamaterial lenses with a slab shape (c) and (d) with a semi-circular shape [13]."

1 W. X. Tang, Z. L. Mei, and T. J. Cui, Sci. China-Phys. Mech. Astron. 58, 127001 (2015). 